\title{
Estructura y biomasa arbórea del Refugio de Vida Silvestre Laquipampa, Lambayeque, Perú
}

\author{
Tree structure and biomass of the Refugio de Vida Silvestre Laquipampa, Lambayeque, Peru \\ Estrutura arbórea e biomassa do Refúgio de Vida Silvestre Laquipampa, Lambayeque, Peru
}

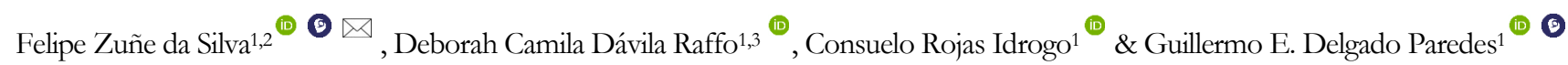

1. Universidad Nacional Pedro Ruiz Gallo, Departamento Académico de Botánica, Lambayeque, Perú

2. Universidade Federal do Rio de Janeiro, Museu Nacional, Programa de Pós-graduação em Ciências Biológicas (Botânica), Rio de Janeiro, Rio de Janeiro, Brasil

3. Universidade Federal do Rio Grande do Sul, Programa de Pós-graduação em Ecologia, Porto Alegre, Rio Grande do Sul, Brasil

Palabras clave:

Bosque tropical estacionalmente seco. Composición arbórea. Suelos. Niveles altitudinales. GLM. Análisis multivariados.

\section{Keywords:}

Seasonally dry tropical forest. Tree composition. Soils. Altitudinal levels. GLM. Multivariate analysis.

\section{Palavras-chave:}

Floresta tropical sazonalmente seca. Composição das árvores. Solos. Niveis altitudinais. GLM. Análise multivariada.

Recebido em: 24/08/2020

Aceito em: 25/03/2021

Editor responsável: Jaílson S. de Novais (UFSB)

eISBN: 2595-6752

\section{(c) (i)}

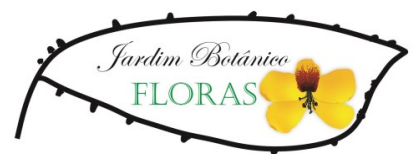

\section{Resumen}

Siendo uno de los ecosistemas tropicales más extensos, el bosque tropical estacionalmente seco es uno de los menos estudiados. El objetivo de este estudio fue determinar la composición florística, estructura y biomasa arbórea del Refugio de Vida Silvestre Laquipampa, así como, describir la influencia de parámetros ambientales. Para ello, se establecieron diez transectos de $50 \times 20 \mathrm{~m}$. En cada transecto fueron evaluados la altura y el diámetro $\geq 10$ $\mathrm{cm})$ de todos los individuos arbóreos. Asimismo, se realizaron colectas botánicas y edáficas. Se aplicaron análisis de agrupamiento, fitosociológicos, multivariados y regresiones. Se registraron 263 individuos, comprendidos en 33 especies, 19 géneros y 17 familias. El área basal total fue de $11,73 \mathrm{~m}^{2} \cdot \mathrm{ha}^{-1}$ y la biomasa promedio de 152,61 t.ha-1. No quedó evidenciado significativamente que la composición florística esté relacionada con las variables edáficas, sin embargo, se observó que estas variables juegan un importante rol en la comprensión de la dinámica del fragmento.

\begin{abstract}
Being one of the most extensive tropical ecosystems, the seasonally dry tropical forest is one of the least studied. This study aims to determine the floristic composition, structure, and tree biomass of the Refugio de Vida Silvestre Laquipampa, as well as to describe the influence of environmental parameters. For this, ten $50 \times 20 \mathrm{~m}$ transects were established. In each transect were evaluated the height and diameter $(\geq 10 \mathrm{~cm})$ of all arboreal individuals. Likewise, botanical, and edaphic collections were made. Clustering, phytosociological, multivariate and regression analyzes were applied. 263 individuals were recorded, comprising 33 species, 19 genera and 17 families. The total basal area was 11.73 $\mathrm{m}^{2} . \mathrm{ha}^{-1}$ and the average biomass of $152.61 \mathrm{t} . \mathrm{ha^{-1 }}$. It was not significantly evidenced that the floristic composition would be related to edaphic variables, however, it was observed that these variables play an important role in the understanding of the fragment dynamics.
\end{abstract}

\section{Resumo}

Apesar de ser um dos ecossistemas tropicais mais extensos, a floresta tropical sazonalmente seca é uma das menos estudadas. O objetivo deste estudo foi determinar a composição florística, estrutura e biomassa arbórea do Refúgio de Vida Silvestre Laquipampa, bem como descrever a influência de seus parâmetros ambientais. Para tal fim, estabeleceu-se dezparcelas de $50 \times 20 \mathrm{~m}$. Em cada parcela foram avaliadas a altura e o diâmetro $(\geq 10 \mathrm{~cm})$ de todos os indivíduos arbóreos. Adicionalmente, realizou-se coletas botânicas e edáficas. Aplicou-se análises de agrupamento, fitosociológicas, multivariadas e de regressão. Registraram-se 263 indivíduos, compreendendo 33 espécies, 19 gêneros e 17 famílias. A área basal total foi de 11,73 $m^{2} . h a^{-1}$ e a biomassa média de 152,61 t.ha $a^{-1}$. Não foi evidenciado significativamente que a composição florística estaria relacionada às variáveis edáficas, entretanto, observou-se que as variáveis ambientais desempenham um papel importante na compreensão dinâmica do fragmento. 


\section{Introducción}

Los bosques tropicales son ecosistemas particularmente esenciales para el funcionamiento de los estándares ecológicos y la subsistencia de la biodiversidad (Sande et al., 2017). Asimismo, juegan un papel indispensable en el ciclo global del carbono terrestre (Houghton, 2005), con depósitos de carbono cuantitativamente significativos almacenados en forma de biomasa (Poorter et al., 2016).

Tales concentraciones se deben en parte al alto índice de riqueza específica por hectárea (van der Sande et al., 2017). Se estima que $\sim 46,500$ especies de árboles se encuentran distribuidos en los trópicos (Slik et al., 2015), por lo que la expresividad en la composición florística (diversidad taxonómica) y en la estructura de las comunidades vegetales podrían estar sujetas a condiciones deterministas de parámetros ambientales (Jones et al., 2016). Sin embargo, tal diversidad se encuentra constantemente amenazada por el aumento de la deforestación, la fragmentación de hábitats y los cambios en el uso de la tierra (Song et al., 2018).

La relevancia de la estructura de las comunidades arbóreas, así como la incorporación de variables de biomasa, suelos, clima y altitud viene siendo cada vez más indispensable para la comprensión de los bosques. Varios trabajos en áreas de pequeña y gran escala reflejan la disposición de estos parámetros en sus estudios (p. ej.; Sanchez et al., 2013; Chave et al., 2014; Rezende et al., 2015; Avitabile et al., 2016). Por tal razón, es que los gradientes ambientales son generalmente señalados como responsables por condicionar los recursos a las comunidades vegetales e influir en las interacciones biológicas (van der Putten et al., 2016).

Con respecto a la biomasa, entre los ecosistemas forestales, el componente arbóreo es soberanamente notable, ya que su articulación retiene grandes cantidades de carbono atmosférico, en comparación con otros tipos de vegetación (Sullivan et al., 2017). Se estima que los árboles tropicales absorben $\sim 72 \mathrm{Pg} \mathrm{C}$ de la atmósfera por año (Beer et al., 2010). Además, se estima que el stock de carbono de los bosques tropicales supera los 370 Pg C (Pan et al., 2011). Estos sumideros de carbono representan un flujo intenso de mitigación continua de uno de los gases de efecto invernadero más importantes, el $\mathrm{CO}_{2}$.

Entre los majestuosos biomas peruanos, se encuentra el Bosque Tropical Estacionalmente Seco (BTES), considerado como unos de los bosques más frágiles y amenazados del mundo (Espinosa et al., 2012; Banda et al., 2016). En la clasificación peruana de los BTES, se destaca los BTES ecuatorianos, que se extienden por las regiones de Piura, Tumbes y Lambayeque, comprendiendo las formaciones de llanura y montaña (Linares-Palomino et al., 2011), destacando las especies arbóreas Prosopis limensis Bentham (algarrobo), Vachellia macracantha (Humb. \& Bonpl. ex Willd.) Seigler \& Ebinger (faique), Capparis angulata (=Colicodendron scabridum (Kunth) Seem.) (zapote), Handroanthus chrysanthus (Jacq.) S.O. Grose, Bombax discolor Kunth y Triplaris guayaquilensis Weed.

En el contexto botánico, diversas exploraciones visando la identificación de la vegetación leñosa de los BTES peruanos han sido reportadas (Linares-Palomino et al., 2011; Delgado-Paredes et al., 2020), aunque no exista una robustez de datos tan grande como en el caso de los bosques húmedos. Sin embargo, en el contexto ecológico, pocos son los estudios que relacionan el componente leñoso con variables ambientales (Rasal et al., 2011; Espinosa et al., 2012), vislumbrando una enorme laguna de conocimiento. Del mismo modo, el área de estudio del presente trabajo carece de información tanto botánica cuanto ecológica, registrando apenas algunos trabajos de ornitología donde mencionan especies vegetales que sirven de alimentos para aves endémicas (Dávila et al., 2019).

En ese aspecto, el presente estudio busca llenar el vacío informativo ecológico referente a la estructura y biomasa arbórea de un área natural protegida, considerando los siguientes cuestionamientos: ¿existe una heterogeneidad del componente arbóreo en el área de estudio? ¿cuál es la cantidad de biomasa presente en estos árboles? Si existiera heterogeneidad, ¿variables ambientales como suelo y altitud, podrían estar influenciando la estructura y biomasa de las comunidades arbóreas? Partiendo de estas interrogantes, se trabajó con la hipótesis de que las variables ambientales jugarían un papel importante sobre las diferencias en la distribución, composición florística, estructura y biomasa arbórea en el área de estudio.

\section{Material y Métodos}

\section{Localización y caracterización del área de estudio}

Este estudio fue desarrollado en el Refugio de Vida Silvestre Laquipampa (RVSL), una de las principales áreas naturales protegidas de la región Lambayeque. Localizado en el distrito de Incahuasi, provincia de Ferreñafe $\left(6^{\circ} 24^{\prime}-6^{\circ} 15^{\prime}\right.$ S y $79^{\circ} 33^{\prime}-79^{\circ} 28^{\prime}$ O) (Figura 1a), el área dispone de 8328,64 ha. Asimismo, presenta un gradiente altitudinal que asciende desde $200 \mathrm{~m}$ en el Bosque estacionalmente seco de llanura (Bes-ll), hasta $2600 \mathrm{~m}$ en el Bosque estacionalmente seco de colina y montaña (Bes-cm), así como, un clima seco, cálido y soleado la mayor parte del año (MINAM, 2019).

\section{Colecta y procesamiento de muestras}

Entre julio y diciembre de 2016 se establecieron de forma asistemática diez transectos de $50 \times 20 \mathrm{~m}$ (Campbell et al., 2002), guardando una distancia mínima de $200 \mathrm{~m}$ entre transectos y distribuidos proporcionalmente en la extensión de cinco pisos altitudinales (Figura 1b, Figura 2). Dentro de cada transecto se registraron datos de altura (estimada a partir de la longitud de la tijera de poda alta) y diámetro de todos los individuos vivos con DAP (diámetro a 1,30 del suelo) $\geq 10 \mathrm{~cm}$. Además, se realizaron colectas botánicas de cada individuo. Posteriormente, las muestras botánicas fueron identificadas a través de bibliografía físico-virtual y consultas a especialistas, clasificándolas de acuerdo con las familias reconocidas por el APG IV (APG, 2016). Finalmente, las muestras fueron herborizadas y depositadas en el Herbario Antenor Orrego (HAO).

Subsecuentemente, fue colectada una muestra de suelo $(\sim 1 \mathrm{Kg})$ por piso altitudinal (entre $20-40 \mathrm{~cm}$ de profundidad). Para los pisos altitudinales que solo contaban con un transecto, las muestras fueron colectadas dentro del mismo transecto. Para los pisos altitudinales que contaban con más de un transecto, las muestras fueron colectadas en un punto intermedio próximo a los transectos. 
Figura 1. Mapa de la región Lambayeque mostrando la ubicación del Refugio de Vida Silvestre Laquipampa (a); ubicación de los transectos en la clasificación altitudinal que presenta el Refugio de Vida Silvestre Laquipampa (b).
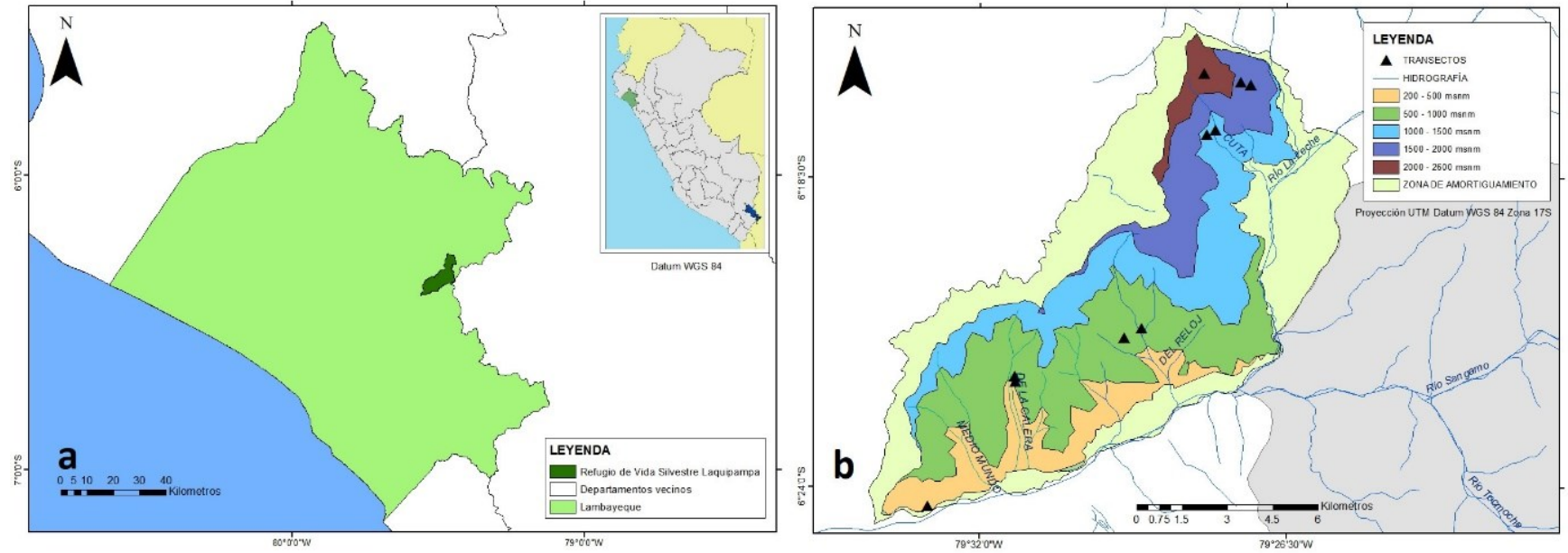

Figura 2. Representación vegetativa de los diferentes pisos altitudinales del Refugio de Vida Silvestre Laquipampa. Vista ascendente-lateral del RVSL (a); Pisos altitudinales: P1 (2000-2600 m) (b), P2 (1500-2000 m) (c), P3 (1000-1500 m) (d), P4 (500-1000 m) (e), P5 (200-500 m) (f).

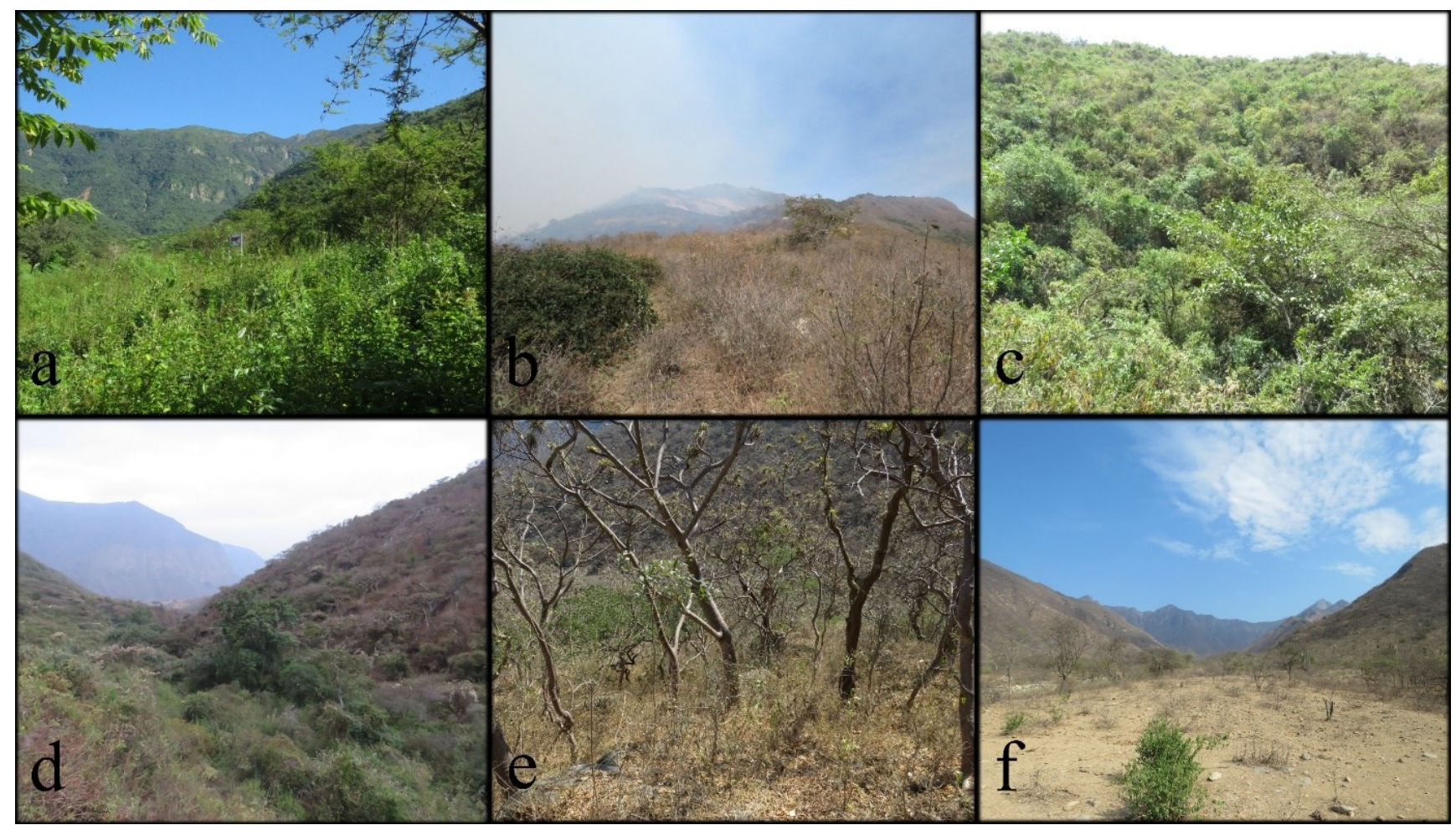

El material fue embalado, etiquetado y enviado al laboratorio de suelos de la Facultad de Agronomía/UNPRG, donde fue procesado y analizado de acuerdo con los protocolos propuestos por el MINAM (2014). Las variables fisicoquímicas medidas fueron: $\mathrm{pH}_{\mathrm{e}}$, conductividad eléctrica $\left(\mathrm{CE}_{\mathrm{e}}\right)$, materia orgánica $(\mathrm{Mo})$, arena (Ao), arcilla (Ar) y limo (Lo). Adicionalmente, se registró como variable la altitud en que se localizaba cada transecto, determinando un total de siete variables ambientales.

\section{Análisis de datos}

Para todos los análisis estadísticos, se adoptó un nivel de significancia del 5\% y 1000 aleatorizaciones para los métodos basados en permutaciones. Cuando pertinentes, las variables en estudio fueron sometidas a análisis de linealidad, normalidad (prueba de Shapiro-Wilk) y homogeneidad de varianzas (prueba de Levene). Cuando no cumplieron con los presupuestos de las pruebas, se sometieron a transformaciones logarítmicas y cuadráticas. Incluso cuando, después de las transformaciones, las variables no cumplieron 
con los presupuestos de las pruebas, se trataron de acuerdo con su distribución inicial. Todos los análisis estadísticos se realizaron en el entorno estadístico R 3.6.3 (R Core Team, 2020) y los análisis de ordenamiento, fitosociológicos y de correlación se realizaron en el programa Fitopac 2.1 (Shepherd, 2010).

Para clasificar los grupos florísticos por piso altitudinal se realizó un análisis UPGMA (Unweighted Pair Group Method with Arithmetic Mean), aplicando el coeficiente de similitud de Jaccard, que otorga igual peso para todas las especies y minimiza posibles problemas de diferencias en el esfuerzo de muestreo (Valentin, 2000). La evaluación de la diversidad alfa se determinó comparando y contrastando los transectos utilizando los índices de ShannonWiener ( $\mathrm{H}^{\prime}$ ) y Pielou $\left(\mathrm{J}^{\prime}\right)$ (Moreno, 2001). Asimismo, se realizó una curva de acumulación de especies utilizando intervalos de confianza del 95\% (Chao et al., 2014). Posteriormente, fue verificada la intensidad y el esfuerzo de muestreo utilizando las fórmulas descritas en Soares et al. (2006), basándose en el número de especies por unidad de muestreo. Para describir asociaciones entre transectos por fisionomía forestal, se realizó un análisis fitosociológico basado en los siguientes parámetros: Dominancia absoluta o área basal (AoD), dominancia relativa (RoD), densidad relativa (RD), frecuencia relativa $(\mathrm{RF})$ y valor de importancia (VI) (Mueller-Dombois; Ellenberg, 2002).

La determinación de la biomasa fue calculada siguiendo patrones alométricos para árboles de bosque tropicales en función del diámetro, altura y densidad de madera, siendo reemplazados en la fórmula de Chave et al. (2014): $\mathrm{B}=0,0673\left(\varrho D^{2} H\right)^{0,976}$, donde, $\mathrm{B}=$ Biomasa (t.ha-1), $\varrho=$ Densidad $\left(\mathrm{g} \cdot \mathrm{cm}^{3}\right), \mathrm{D}=$ Diámetro $(\mathrm{cm})$ y $\mathrm{H}=$ Altura (m). Para la densidad se utilizó la base de datos presente en The World Agroforestry Centre (www.worldagroforestry.org). Cuando no se obtuvo datos a nivel de densidad específica, se procedió a utilizar resultados a nivel de género o familia. Cuando no se logró obtener ningún dato se usó una media global de 0,62 g.cm³ (Chave et al., 2009). Consecuentemente, se compararon los valores medios de biomasa entre familias, transectos y pisos altitudinales.

Para determinar si las variables ambientales explicarían la estructura (área basal, altura promedio por transecto), composición florística (número de especies por transecto) y biomasa arbórea, se aplicó un modelo lineal generalizado (GLM), aplicando distribución gaussiana (con 'identity link') para la estructura y biomasa y, distribución de Poisson (com 'log link') para la composición florística. Además, se aplicó un análisis de desviación de varianza para la comparación entre modelos (Sakate; Kashid, 2014), y se seleccionaron aquellos modelos con los valores más bajos según el criterio AIC (Burnham et al., 2011). Posteriormente, se filtraron las variables ambientales a partir de los GLMs, donde se seleccionó la biomasa y aquellas variables ambientales que mejor explicaron los modelos, y en conjunto con la composición florística se aplicó un Análisis de Correspondencia Canónica (CCA), ejecutando la prueba de Monte Carlo para verificar la significancia de los ejes de la ordenación (ter Braak; Prentice, 1988). Finalmente, se probó la correlación entre la disimilaridad florística y ambiental, usando distancia euclidiana y aplicando la prueba parcial de Mantel (rM), que tuvo como finalidad la determinación de la distancia entre la composición florística y ambiental, teniendo como hipótesis nula la ausencia de correlación linear entre las mismas (Legendre; Legendre, 2012).

\section{Resultados y discusión}

Fueron registrados 263 individuos comprendidos en 33 especies, 19 géneros y 17 familias. Alrededor del 50\% de las especies colectadas durante la época en que se realizaron los muestreos no presentaron estructuras fértiles, razón por la cual algunos individuos no fueron identificados a nivel infragenérico, incluso, en casos excepcionales la identificación no fue posible a nivel de familia. Al respecto, LealPinedo y Linares-Palomino (2005), sugieren que para la obtención de datos florísticos precisos sería necesario el marcaje de los individuos y una mayor inversión de tiempo para realizar visitas y colectas periódicas intensivas.

El análisis de agrupación demostró tener una alta correlación cofenética $(0,86)$ entre pisos altitudinales, observándose una menor disparidad entre los pisos altitudinales P4 y P5 con 25\% de similitud (Figura 3), dejando a los demás pisos sobre los 15\%. Debido a la irregularidad en el número de especies por piso altitudinal, se optó por seleccionar una especie indicadora por piso de acuerdo con el mayor valor de abundancia (Figura 3), no llegando a ser un análisis de especies indicadoras como propone Dufrêne y Legendre (1997), ya que estos no presentan valores indicadores ni significancia.

Siguiendo la lógica del número de individuos, las familias que presentaron mayores valores fueron: Fabaceae (66), Malvaceae (42) y Anacardiaceae (41), en tanto que los géneros con mayor número de individuos en cada una de estas familias fueron Caesalpinia L. (34), Eriotheca Schott \& Endl. (42) y Loxopterygium Hook. f. (41), respectivamente. Loxopterygium huasango Spruce ex Engl. (36) y Eriotheca mizii Schott \& Endl. (40), fueron las especies con mayor número de individuos, mientras que Caesalpinia spinosa (Molina) Kuntze (33 individuos) fue la única especie registrada solamente en un transecto (T1) y, por ende, en solo un piso altitudinal (P1).

La curva de acumulación de especies mostró diferencias entre las especies observadas y estimadas para el área de estudio (Figura 4); sin embargo, no llegó a estabilizarse integralmente, sugiriendo que el aumento en el número de transectos podría indicar un mayor número de especies. Consecuentemente, la intensidad de muestreo fue de $0,01 \%$, resaltando a través del esfuerzo de muestreo la necesidad de acrecentar 262 transectos más para obtener la estabilidad en el número de especies. Tal expresividad en la inferencia adicional del número de unidades muestrales refuerza la necesidad de un intenso levantamiento florístico y de la determinación especifica de los taxones (Leal-Pinedo; Linares-Palomino, 2005; Soares et al., 2006). La diversidad varió entre 0,79-2,45 (H') y la equidad entre 0,72-0,96 (J'). En cuanto al número de especies por familia se destacó Fabaceae con diez especies seguida de Myrtaceae con tres y Capparaceae y Rosaceae con solamente dos especies. Varios estudios pioneros realizados por Weberbauer (1945) y Ferreyra (1983), enfatizaron sobre la gran diversidad de especies leñosas en los bosques tropicales estacionalmente secos de Lambayeque y el norte del Perú, donde se formaban varias asociaciones de especies leñosas. Respecto a los índices de Shannon- 
Figura 3. Agrupamiento por similitud de Jaccard realizado para los diferentes pisos altitudinales del Refugio de Vida Silvestre Laquipampa, Lambayeque, Perú. $\mathrm{P}=$ Piso altitudinal; $\mathrm{T}$ = Transecto; Número de individuos por especie (entre paréntesis).

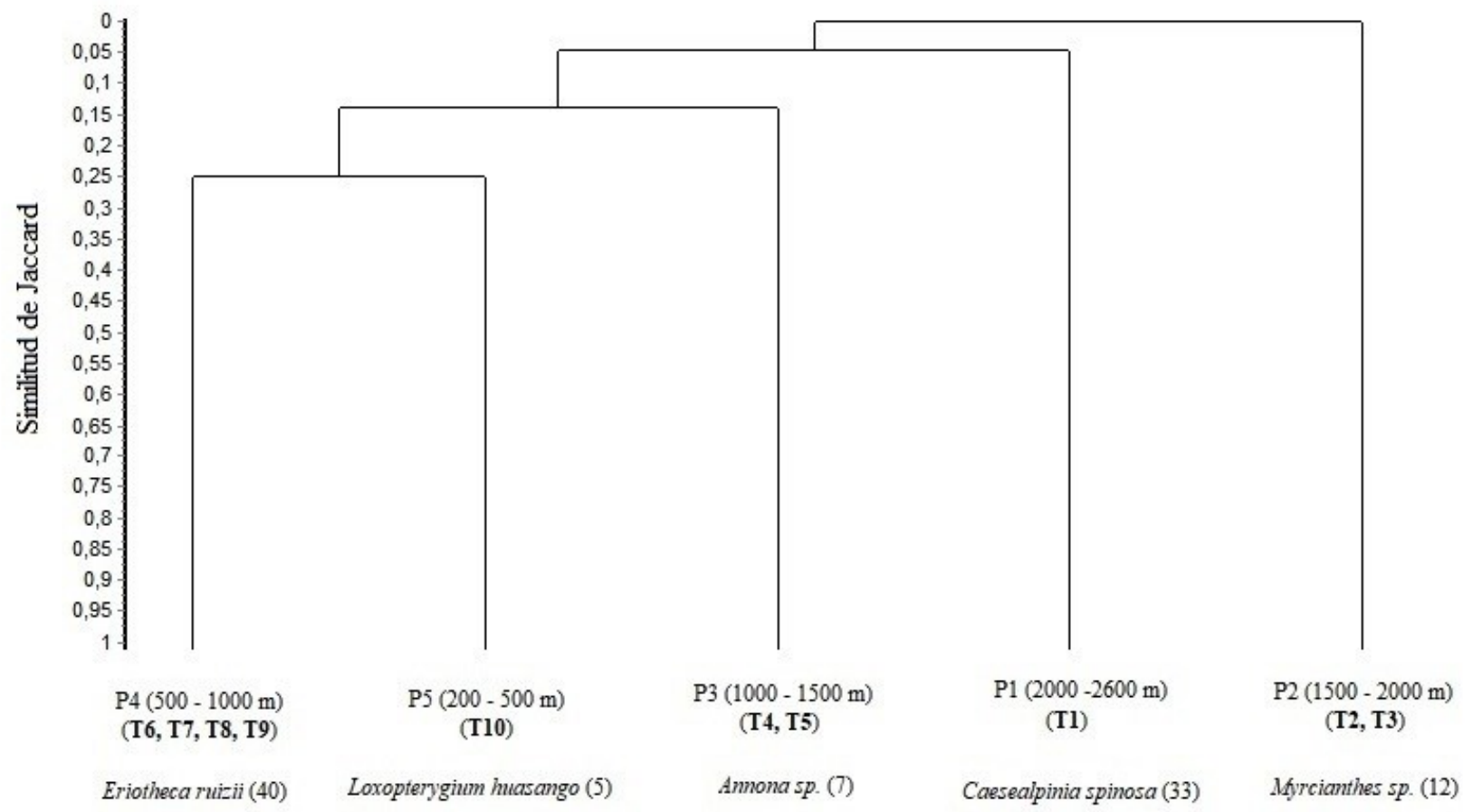

Figura 4. Curva de acumulación de especies para la riqueza observada y estimada del Refugio de Vida Silvestre Laquipampa, Lambayeque, Perú.

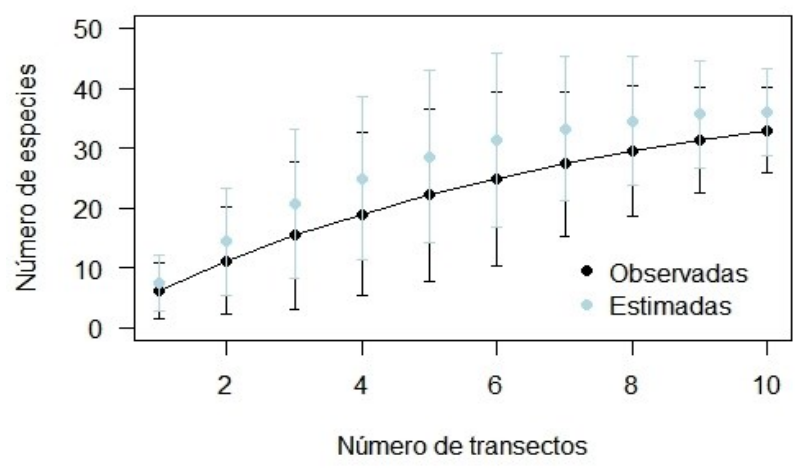

Wiener, en el presente estudio se evidenciaron valores intermedios en comparación con los BTES de La Menta y Timbes $(1,17-2,20)$ (Rasal et al., 2011) y los BTES de Loja (2,38-2,82) (Aguirre et al., 2013). En cuanto al índice de equidad de Pielou, este arrojó valores superiores a los BTES de Loja $(0,64-0,67)$.

Os registros de los parámetros fitosociológicos señalaron un área basal de $11,73 \mathrm{~m}^{2} \cdot \mathrm{ha}^{-1}$, siendo las familias Fabaceae y Malvaceae las de mayores extensiones con 2,80 y $1,93 \mathrm{~m}^{2} \cdot \mathrm{ha}^{-1}$, respectivamente (Tabla 1). En el estudio realizado por Alva-Mendonza et al. (2020), se reportó un área basal de 26,1 $\mathrm{m}^{2} \cdot \mathrm{ha}^{-1}$ para la vegetación del Bosque de Protección de Pagaibamba; en tanto que, Rasal-Sanchez et al. (2012) reportaron $89,23 \mathrm{~m}^{2} \cdot \mathrm{ha}^{-1}$ para el bosque montano de Lanchurán (Piura), ambos bosques pertenecientes a los BTES peruanos. Asimismo, los valores de RD y RF fueron mayores en las familias Fabaceae, Malvaceae y Anacardiaceae, los cuales se correlacionan con sus respectivos VIF de $64,56,44,89$ y 41,39. Por otro lado, los VIE corresponden a las familias Malvaceae, Anacardiaceae y Burseraceae, destacadas por sus respectivas especies: Eriotheca ruizii (42,89), Loxopterygium huasango $(39,46)$ y Bursera graveolens (Kunth) Triana \& Planch. $(29,82)$ (Tabla 1). En general, la dinámica del bosque ha sido muy acelerada en los últimos años puesto que para Gentry (1995) las familias Fabaceae y Bignoniaceae tendían a dominar los BTES; sin embargo, Marcelo-Peña (2008), reportaron que las familias dominantes fueron Boraginaceae $y$ Malvaceae, mientras que en el estudio de Espinosa et al. (2012) la familia dominante fue Fabaceae, concordando con lo reportado en este trabajo.

En cuanto a los cálculos dendrométricos se observó que los transectos T10 $(27,80 \mathrm{~cm})$ y T9 $(21,80 \mathrm{~cm})$, pertenecientes al P5 y $\mathrm{P} 4$, respectivamente, presentaron los mayores valores de mediana para diámetro, mientras que los menores valores fueron representados por los transectos T3 $(13,05 \mathrm{~cm})$ y T2 $(12,50 \mathrm{~cm})$, referentes al P2 (Tabla 2). Por otro lado, el mayor valor medio de altura se concentró en el transecto T10 $(9,98 \mathrm{~m})$, en tanto que el menor valor se registró en el transecto T1 (5,67 m), referente al P1 (Tabla 2). Tanto la mediana de los diámetros cuanto la altura promedio de los transectos se encontraron en el intermedio de valores extremos reportados en los trabajos de Rasal et al. (2011), Alva-Mendonza et al. (2020) y Delgado-Paredes et al. (2020).

La estimativa promedio de biomasa fue 152,61 \pm 73,81 t.ha-1, con valores extremos de 57,96 \pm 45,50 t.ha-1 ${ }^{-1}$, en el T3, referente al P2 y 345,82 $\pm 245,61$ t.ha ${ }^{-1}$ en el T10, referente al P5 (Tabla 2). Por otro lado, el promedio de individuos por familia con el mayor aporte de biomasa fue de Moraceae $\left(618,47 \pm 330,44\right.$ t.ha $\left.{ }^{-1}\right)$, seguida de las familias Lauraceae $\left(404,03 \pm 363,78\right.$ t.ha $\left.^{-1}\right)$, Anacardiaceae 
Tabla 1. Parámetros fitosociológicos de las especies muestreadas en el Refugio de Vida Silvestre Laquipampa, Lambayeque, Perú. Esp. = especies; Ind. = individuos; $\mathrm{RD}=$ dominancia relativa $(\%) ; \mathrm{RF}=$ frecuencia relativa $(\%) ;$ AoD $=$ dominancia absoluta $\left(\right.$ área basa; $\mathrm{m}^{2}$. ha $\left.{ }^{-1}\right) ; \mathrm{RoD}=\mathrm{dominancia}$ relativa $(\%)$; $\mathrm{VIE}=$ valor de importancia por especie; VIF = valor de importancia por familia.

\begin{tabular}{|c|c|c|c|c|c|c|c|c|}
\hline Familia/Especie & No Esp. & No Ind. & RD & $\mathbf{R F}$ & AoD & RoD & VIE & VIF \\
\hline \multicolumn{9}{|l|}{ ANACARDIACEAE } \\
\hline Loxopterygium buasango & & 41 & 15,59 & 8,06 & 1,61 & 15,8 & 39,46 & \\
\hline Total Familia & 1 & 41 & 15,59 & 8,06 & 1,61 & 15,8 & 39,46 & 41,39 \\
\hline \multicolumn{9}{|l|}{ ANNONACEAE } \\
\hline Annona sp. & & 7 & 2,66 & 1,61 & 0,17 & 1,71 & 5,99 & \\
\hline Total Familia & 1 & 7 & 2,66 & 1,61 & 0,17 & 1,71 & 5,99 & 6,37 \\
\hline \multicolumn{9}{|l|}{ ARALIACEAE } \\
\hline Oreopanax sp. & & 4 & 1,52 & 1,61 & 1,61 & 0,69 & 3,83 & \\
\hline Total Familia & 1 & 4 & 1,52 & 1,61 & 1,61 & 0,69 & 3,83 & 4,22 \\
\hline \multicolumn{9}{|l|}{ BURSERACEAE } \\
\hline Bursera graveolens & & 29 & 11,03 & 9,68 & 0,93 & 9,12 & 29,82 & \\
\hline Total Familia & 1 & 29 & 11,03 & 9,68 & 0,93 & 9,12 & 29,82 & 32,15 \\
\hline \multicolumn{9}{|l|}{ CAPPARACEAE } \\
\hline Capparis scabrida & & 5 & 1,90 & 4,84 & 0,10 & 1,0 & 7,74 & \\
\hline C. mollis & & 1 & 0,38 & 1,61 & 0,04 & 0,36 & 2,35 & \\
\hline Total Familia & 2 & 6 & 2,28 & 6,45 & 0,14 & 1,36 & 10,09 & 11,64 \\
\hline \multicolumn{9}{|l|}{ FABACEAE } \\
\hline Albizia multiflora & & 1 & 0,38 & 1,61 & 0,03 & 0,25 & 2,25 & \\
\hline Caesalpinia spinosa & & 34 & 12,93 & 3,23 & 1,36 & 13,43 & 29,56 & \\
\hline Erytbrina smitbiana & & 10 & 3,80 & 1,61 & 0,67 & 6,57 & 11,98 & \\
\hline Fabaceae sp. 1 & & 2 & 0,76 & 1,61 & 0,02 & 0,16 & 2,54 & \\
\hline Fabaceae sp. 2 & & 2 & 0,76 & 3,23 & 0,05 & 0,48 & 4,47 & \\
\hline Fabaceae sp. 3 & & 1 & 0,38 & 1,61 & 0,02 & 0,17 & 2,17 & \\
\hline Fabaceae sp. 4 & & 1 & 0,38 & 1,61 & 0,01 & 0,09 & 2,08 & \\
\hline Inga sp. 1 & & 6 & 2,28 & 1,61 & 0,40 & 3,92 & 7,81 & \\
\hline Inga sp. 2 & & 4 & 1,52 & 1,61 & 0,10 & 1,02 & 4,15 & \\
\hline Vachellia macracantha & & 5 & 1,9 & 4,84 & 0,14 & 1,4 & 8,14 & \\
\hline Total Familia & 10 & 66 & 25,09 & 22,57 & 2,80 & 27,49 & 75,15 & 64,56 \\
\hline \multicolumn{9}{|l|}{ GROSSULARIACEAE } \\
\hline Escallonia micrantha & & 2 & 0,76 & 3,23 & 0,04 & 0,36 & 4,34 & \\
\hline Total Familia & 1 & 2 & 0,76 & 3,23 & 0,04 & 0,36 & 4,34 & 5,12 \\
\hline HYPERICACEAE & & & & & & & & \\
\hline Hypericaceae & & 1 & 0,38 & 1,61 & 0,00 & 0,00 & 1,99 & \\
\hline Total Familia & 1 & 1 & 0,38 & 1,61 & 0,00 & 0,00 & 1,99 & 2,38 \\
\hline LAURACEAE & & & & & & & & \\
\hline Beilschmiedia sulcata & & 6 & 2,28 & 3,23 & 0,53 & 5,17 & 10,67 & \\
\hline Total Familia & 1 & 6 & 2,28 & 3,23 & 0,53 & 5,17 & 10,67 & 11,45 \\
\hline MALVACEAE & & & & & & & & \\
\hline Eriotheca ruizii & & 42 & 15,97 & 8,06 & 1,93 & 18,92 & 42,96 & \\
\hline Total Familia & 1 & 42 & 15,97 & 8,06 & 1,93 & 18,92 & 42,96 & 44,89 \\
\hline MORACEAE & & & & & & & & \\
\hline Ficus cuatrecasasiana & & 6 & 2,28 & 3,23 & 1,16 & 11,39 & 16,89 & \\
\hline Total Familia & 1 & 6 & 2,28 & 3,23 & 1,16 & 11,39 & 16,89 & 17,67 \\
\hline MYRTACEAE & & & & & & & & \\
\hline Myrcia mollis & & 3 & 1,14 & 3,23 & 0,04 & 0,38 & 4,74 & \\
\hline Myrcia sp. & & 7 & 2,66 & 3,23 & 0,12 & 0,13 & 7,02 & \\
\hline Myrcianthes sp. & & 12 & 4,56 & 3,23 & 0,19 & 1,83 & 9,62 & \\
\hline Total Familia & 3 & 22 & 8,36 & 9,69 & 0,35 & 2,34 & 21,38 & 19,71 \\
\hline NN1 & & & & & & & & \\
\hline NN1 & & 2 & 0,76 & 1,61 & 0,02 & 0,23 & 2,6 & \\
\hline Total Familia & 1 & 2 & 0,76 & 1,61 & 0,02 & 0,23 & 2,6 & 2,99 \\
\hline NN2 & & & & & & & & \\
\hline NN2 & & 1 & 0,38 & 1,61 & 0,02 & 0,23 & 2,22 & \\
\hline Total Familia & 1 & 1 & 0,38 & 1,61 & 0,02 & 0,23 & 2,22 & 2,61 \\
\hline NN3 & & & & & & & & \\
\hline NN3 & & 2 & 0,76 & 1,61 & 0,04 & 0,43 & 2,8 & \\
\hline Total Familia & 1 & 2 & 0,76 & 1,61 & 0,04 & 0,43 & 2,8 & 3,19 \\
\hline POLYGONACEAE & & & & & & & & \\
\hline Coccoloba gracilis & & 2 & 0,76 & 3,23 & 0,06 & 0,58 & 4,57 & \\
\hline Total Familia & 1 & 2 & 0,76 & 3,23 & 0,06 & 0,58 & 4,57 & 5,34 \\
\hline ROSACEAE & & & & & & & & \\
\hline Rosaceae sp. 1 & & 9 & 3,42 & 3,23 & 0,09 & 0,88 & 7,52 & \\
\hline Rosaceae sp. 2 & & 8 & 3,04 & 3,23 & 0,14 & 1,42 & 7,69 & \\
\hline Total Familia & 2 & 17 & 6,46 & 6,46 & 0,23 & 2,3 & 15,21 & 12,76 \\
\hline RUBIACEAE & & & & & & & & \\
\hline Rubiaceae sp. & & 3 & 1,14 & 1,61 & 0,04 & 0,41 & 3,16 & \\
\hline Total Familia & 1 & 3 & 1,14 & 1,61 & 0,04 & 0,41 & 3,16 & 3,55 \\
\hline RUTACEAE & & & & & & & & \\
\hline Zanthoxylum sp. & & 3 & 1,14 & 3,23 & 0,04 & 0,37 & 4,74 & \\
\hline Total Familia & 1 & 3 & 1.14 & 3,23 & 0,04 & 0,37 & 4,74 & 5,51 \\
\hline SAPINDACEAE & & & & & & & & \\
\hline Serjania sp. & & 1 & 0,38 & 1,61 & 0,01 & 0,13 & 2,12 & \\
\hline Total Familia & 1 & 1 & 0,38 & 1,61 & 0,01 & 0,13 & 2,12 & 2,51 \\
\hline Total & 33 & 263 & 100 & 100 & 11,73 & 100 & 300 & 300 \\
\hline
\end{tabular}


$\left(198,85 \pm 152,17\right.$ t.ha $\left.^{-1}\right)$, Fabaceae $(149,51 \pm 227,08$ t.ha-1 $)$ y Malvaceae $\left(140,71 \pm 183,75\right.$ t.ha $\left.^{-1}\right)$, en tanto que otras familias mostraron valores inferiores a 80 t.ha $^{-1}$ (Figura 5). El elevado valor de biomasa para la familia Moraceae se debe a un caso excepcional en que uno de los seis individuos de Ficus cuatrecasasiana Dugand (única especie muestreada de la familia) presentó el mayor valor diamétrico $(93,13$ $\mathrm{cm}$ ) entre todos los 263 individuos muestreados. De igual manera, uno de los seis individuos de la especie Beilschmiedia sulcata (Ruiz \& Pav.) Kosterm. (única representante de la familia Lauraceae), presentó un diámetro de 56,61 cm y altura de $16 \mathrm{~m}$, lo que contribuyó para el incremento de biomasa promedio de la familia. Otras familias también presentaron casos aislados de individuos con valores extremos (outliers); sin embargo, el número de individuos por familia no permitió un marcado incremento promedio de biomasa (Figura 5).

Comparando los resultados de este trabajo con los realizados en ambientes similares o aledaños a nuestra área de estudio, Málaga et al. (2014), mediante la estimación de biomasa por ecozonas del país, determinaron 17,09 tha ${ }^{-1}$ para la costa. Asimismo, Díaz et al. (2015), calcularon que la cantidad de carbono fijada en el Santuario Histórico Bosque de Pómac fue 255,09 t.ha-1. Por su parte, Asner et al. (2014), en un informe conjunto del Observatorio Aéreo Carnegie y el MINAM, reportaron sobre la densidad de carbono en las Áreas Naturales Protegidas, indicando que la media de biomasa para el Refugio de Vida Silvestre

Figura 5. Estimativa de biomasa de individuos por familia en el Refugio de Vida Silvestre Laquipampa, Lambayeque, Perú. $*=$ familias no identificadas.

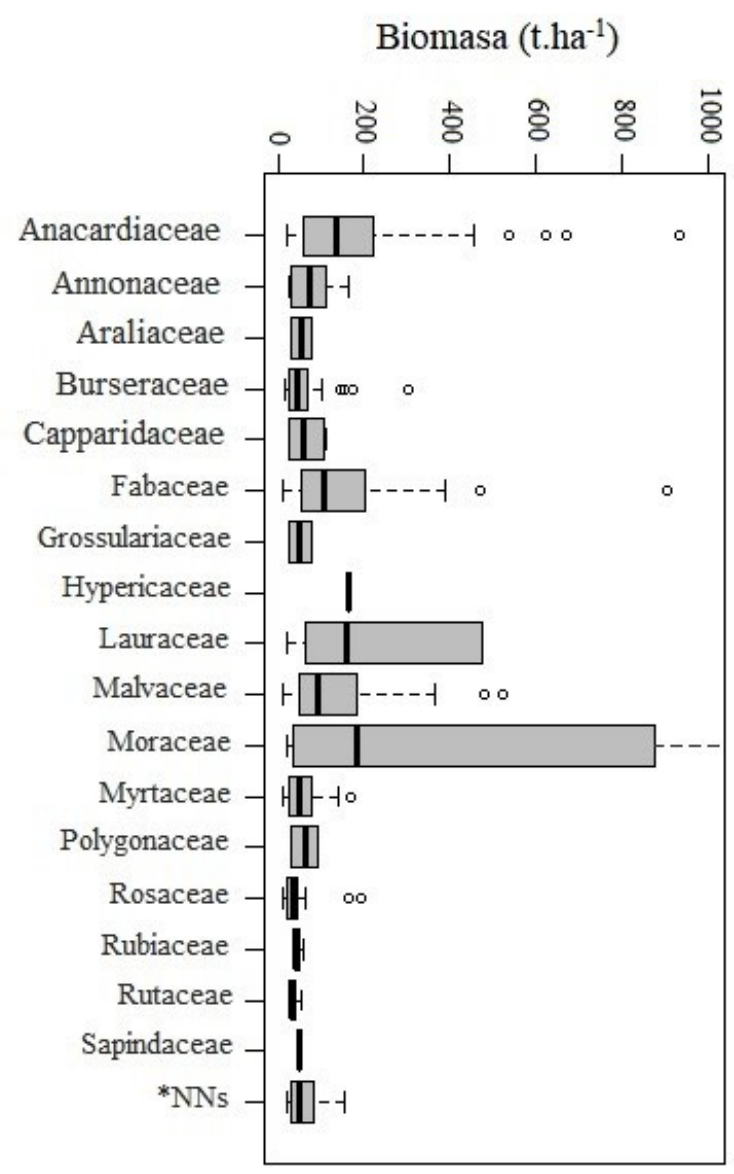

Laquipampa fue 4 t.ha-1 ${ }^{-1}$ contrastando notablemente con los de este estudio. Sin embargo, cabe destacar que la información registrada por el MINAM mostró una incertidumbre de hasta $80 \%$, evidenciando un muestreo no representativo.

Por otro lado, investigaciones realizadas a nivel sudamericano y en diferentes tipos de bosques contrastaron con la nuestra debido a que utilizaron modificaciones metodológicas. Es así que, en varios estudios realizados en la cuenca amazónica, como el de Tello y Flores (2010), determinaron 313,22 t.ha-1; asimismo, Pallqui et al. (2014), en un estudio similar estimaron un promedio de 296,20 tha1. Adicionalmente, en bosques con características climatológicas similares al nuestro, se encontró en un área privada en la caatinga de Piauí (Brasil) un total de 170 tha $^{-1}$ (Brand, 2017), en tanto que estudios realizados en Ecuador, revelaron que, para bosques secundarios, rurales y forestales, la biomasa fue estimada en 223 t.ha ${ }^{-1}$ (López et al., 2002), 9,57 t.ha ${ }^{-1}$ (Ordoñez et al., 2007) y 565,44 t.ha-1 (Cueva et al., 2019), respectivamente.

Se observó que la conductividad eléctrica estuvo por debajo de 0,91, clasificándose como suelos no salinos. En cuanto al $\mathrm{pH}$ y Mo, todos los pisos fueron clasificados como neutros y con alto contenido de materia orgánica (Tabla 3). Tales resultados reflejan el padrón característico de la región Lambayeque (GRL, 2012), asî como, de otros bosques estacionalmente secos del norte del Perú.

Tabla 2. Medianas de diámetros, medias de alturas y medias estimadas de biomasa por transectos y piso altitudinal del Refugio de vida Silvestre Laquipampa, Lambayeque, Perú. $\mathrm{T}=$ transecto; $\pm=$ desviación estándar.

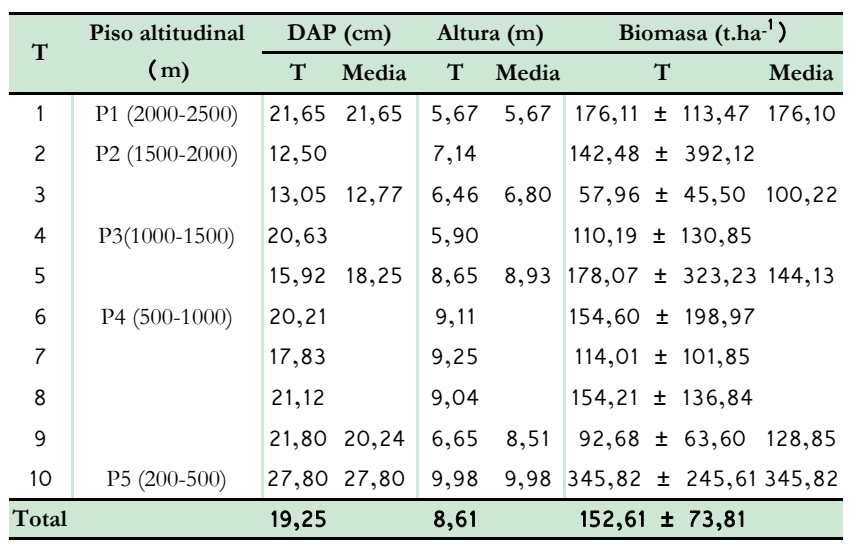

Tabla 3. Clasificación de variables edafológicas por piso altitudinal muestreadas en el Refugio de Vida Silvestre Laquipampa, Lambayeque, Perú.

\begin{tabular}{|c|c|c|c|c|c|}
\hline \multirow{4}{*}{$\begin{array}{l}\text { Variables } \\
\text { edafológicas }\end{array}$} & \multicolumn{5}{|c|}{ Piso altitudinal (m ) } \\
\hline & $\begin{array}{c}\text { P1 (2000- } \\
2600)\end{array}$ & $\begin{array}{c}\text { P2 (1500- } \\
2000)\end{array}$ & $\begin{array}{c}\text { P3 (1000- } \\
1500)\end{array}$ & $\begin{array}{l}\text { P4 (500- } \\
1000)\end{array}$ & $\begin{array}{c}\text { P5 (200 } \\
500)\end{array}$ \\
\hline & \multicolumn{5}{|c|}{ Clases texturales } \\
\hline & $\begin{array}{l}\text { Franco- } \\
\text { arcillo- } \\
\text { arenoso }\end{array}$ & Arcilloso & Arenoso & $\begin{array}{l}\text { Franco } \\
\text { arcillo- } \\
\text { arenoso }\end{array}$ & $\begin{array}{l}\text { Franco- } \\
\text { arenoso }\end{array}$ \\
\hline Ao & 47,84 & 35,98 & 52,13 & 49,98 & 63,70 \\
\hline Ar & 29,04 & 43,04 & 17,04 & 24,04 & 18,72 \\
\hline Lo & 23,12 & 20,97 & 30,83 & 25,97 & 17,58 \\
\hline $\mathrm{CE}_{\mathrm{e}}$ & 0,30 & 0,29 & 0,75 & 0,91 & 0,42 \\
\hline $\mathrm{pH}_{\mathrm{e}}$ & 6,90 & 7,00 & 6,70 & 7,10 & 6,80 \\
\hline Mo & 7,00 & 5,00 & 7,60 & 5,00 & 6,60 \\
\hline
\end{tabular}


Tal y como representan Rasal et al. (2011) en su estudio, los bosques de La Menta y Timbes poseen un suelo con $\mathrm{pH}$ neutro, conductividad eléctrica no salina, y textura franco-arcillosa, resultados parecidos al obtenido en este estudio, discrepando solamente por el porcentaje de materia orgánica.

Nuestros mejores GLMs mostraron que la composición florística de los transectos estuvo fuertemente relacionada al $\mathrm{pH}$ del suelo, mientras que el área basal y la biomasa estuvieron asociadas a la arcilla y al limo (Tabla 4). De igual manera, en un estudio realizado en matorrales enanos se encontró que la riqueza y composición de especies están más ligadas al pH del suelo que a cualquier otra variable ambiental o de suelo (Chytrý et al., 2010). Por otro lado, la altura media de los individuos por transecto no tuvo relación con la textura del suelo y sí con la conductividad. Al respecto, Muenchow et al. (2013), determinó que, para los bosques estacionalmente secos del norte del Perú, las variables edáficas fueron más importante en la estructuración de la vegetación que otras variables ambientales, como precipitación media anual.

El diagrama de ordenación del CCA reveló un gradiente más relacionado al eje 1 para las especies presentes en el T4 y T5, referentes al P3, mientras que el eje 2 estuvo más asociado al T1, referente al P1 (Figura 6). La variable ambiental $\mathrm{pH}(0,94)$ estuvo fuertemente relacionada al eje 1 , mientras que las variables de altitud $(-0,74)$ y conductividad eléctrica $(0,60)$ estuvieron más relacionada al eje 2 . Los autovalores (AV) canónicos obtenidos para los dos primeros ejes de la ordenación fueron 0,88 y 0,96 , siendo responsables, respectivamente por $22,41 \%$ y $24,52 \%$ de la varianza acumulada de los datos. Tal y como determinaron Rasal et al. (2011), las variables edafologías podrían variar a lo largo de un gradiente altitudinal con variación en la composición edáfica, confirmado por Sánchez et al. (2013) y Delgado-Paredes et al. (2020), aunque estos últimos no realizaron análisis de suelo. Asimismo, Cueva et al. (2019), observaron que la altitud, al contrario de otras variables, podría estar fuertemente relacionada con la estructura y biomasa arbórea en bosques tropicales estacionalmente secos.
Tabla 4. Modelos lineares generalizados elaborados a partir de la relación entre variables respuestas y predictoras. Significancia

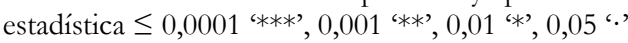

\begin{tabular}{|c|c|c|c|c|c|}
\hline \multicolumn{6}{|c|}{ Modelo $=$ Composición florística $\sim \mathrm{Mo}+\mathrm{pH}$} \\
\hline Variable & Estimado & Error Est. & Desviación & Desv. Resid. & $\mathrm{p}(>$ Chi $)$ \\
\hline Nulo & & & & 23,52 & \\
\hline Mo & $-1,55$ & 0,35 & 1,03 & 22,49 & 0,3 \\
\hline $\mathrm{pH}$ & $-11,44$ & 2,68 & 19,49 & 2,99 & $0,0001^{* * *}$ \\
\hline \multicolumn{6}{|c|}{ Modelo $=$ Área basal $\sim$ Ar + Lo } \\
\hline Variable & Estimado & Error Est. & Desviación & Desv. Resid. & $\mathrm{p}(>\mathrm{Chi})$ \\
\hline Nulo & & & & 0,0011 & \\
\hline Ar & $-0,0012$ & 0,0002 & 0,0005 & 0,0006 & $0,0001^{* * *}$ \\
\hline Lo & $-0,0019$ & 0,0005 & 0,0004 & 0,0001 & $0,0001^{* * *}$ \\
\hline \multicolumn{6}{|c|}{ Modelo $=$ Altura media $\sim \mathrm{CE}_{\mathrm{e}}+$ Lo } \\
\hline Variable & Estimado & Error Est. & Desviación & Resid. Dev. & $\mathrm{p}(>\mathrm{Chi})$ \\
\hline Nulo & & & & 22,52 & \\
\hline $\mathrm{CE}_{\mathrm{e}}$ & 5,04 & 2,14 & 9,79 & 12,38 & $0,01^{*}$ \\
\hline Lo & $-0,27$ & 0,14 & 0,34 & 22,18 & 0,65 \\
\hline \multicolumn{6}{|c|}{ Modelo $=$ Biomasa $\sim$ Ar + Lo } \\
\hline Variable & Estimado & Error Est. & Desviación & Resid. Dev. & $\mathrm{p}(>\mathrm{F})$ \\
\hline Nulo & & & & 54479 & \\
\hline $\operatorname{Ar}$ & $-7,57$ & 1,74 & 32730 & 12187 & 0,05 \\
\hline Lo & $-10,01$ & 3,91 & 9563 & 44917 & $0,003^{* *}$ \\
\hline
\end{tabular}

El resultado de la prueba de Monte Carlo fue significativo para los dos ejes de la ordenación (AV1 p = 0,05; AV2 p = 0,003). La hipótesis de que la composición florística estaría determinada por las variables ambientales, usando la prueba de Mantel, no fue confirmada ( $\mathrm{rM}=0,06 ; \mathrm{p}=0,36)$. En ese sentido, para un mejor acercamiento de los resultados a la hipótesis establecida, sería necesario tanto una mayor solidez de datos florísticos cuanto un mayor volumen de filtros ambientales, los cuales podrían divergir de nuestros resultados. Finalmente, cabe resaltar que, si se llegara a identificar todos los taxones presentes en nuestro estudio, una nueva serie de análisis sería lo suficientemente necesario para concebir una mejor comprensión ecológica del área de estudio.

Figura 6. Diagrama de ordenación de los ejes del Análisis de Correlación Canónica (CCA) de la composición florística y ambiental del Refugio de Vida Silvestre Laquipampa, Lambayeque, Perú. T = Transecto. $\mathrm{P}=$ Pisos altitudinales.

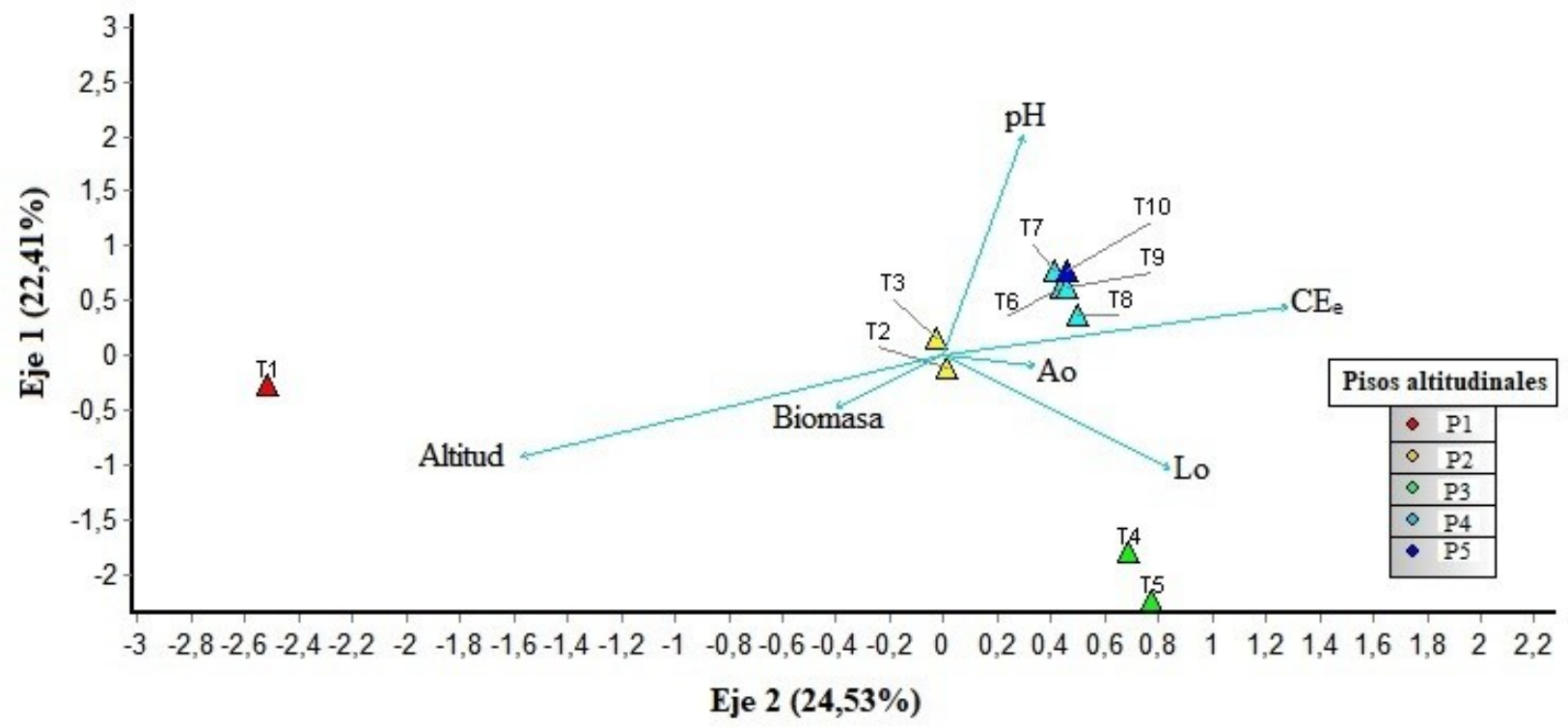




\section{Conclusión}

El componente leñoso del Refugio de Vida Silvestre Laquipampa presenta una heterogeneidad taxonómica que está influenciada por patrones estructurales y atributos físico-ambientales. La composición florística del fragmento presentó familias y especies representativas de Bosques Tropicales Estacionalmente Secos peruanos, por lo que el área de estudio sería un fiel reflejo de las áreas naturales protegidas de Lambayeque. Asimismo, la biomasa arbórea demostró altos valores en sus almacenamientos, siendo influenciados por individuos aislados de gran porte. Aunque no quedó evidenciado significativamente que la composición florística estuvo estrechamente relacionada con variables edáficas, se observó que estas variables serían importantes para la comprensión estructural del fragmento.

\section{Agradecimientos}

Se agradece por el apoyo en campo a Alejandro y Napoleón, guardaparques del RVSL. De modo particular a William Martínez, jefe del RVSL, por el respaldo logístico. También, a Alejandro Vásquez y Wendy Herrera, estudiantes de la UNPRG, por el apoyo técnico. Finalmente, a Segundo Leiva y Luis Chang por recibir nuestras muestras botánicas en el HAO.

\section{Financiamiento}

Los autores declaran que no existen fuentes de financiación para informar.

\section{Contribuciones autorales}

Conceptualización: FZD, DCDR. Conservación de datos: FZD, DCDR. Análisis formal: FZD, DCDR. Investigación: FZD, DCDR. Metodología: FZD, DCDR. Supervisión: CRI, GDP. Validación: GDP. Visualización: CRI, GDP. Redacción - borrador original: FZD, DCDR. Redacción - revisión y edición: FZD, DCDR, CRI, GDP.

\section{Conflicto de interés}

Los autores declaran que no existen conflictos de intereses que informar.

\section{Disponibilidad de datos}

Los datos completos analizados durante el estudio actual se pueden proporcionar a petición justificada al autor para correspondencia.

\section{Conformidad ética}

No se aplica.

\section{Referencias}

Aguirre Z, Betancourt-Figueras Y, Geada-López G. Regeneración natural en los bosques secos de la provincia de Loja y utilidad para el manejo local. Revista Cedamaz 2013;3(1):54-65.

Alva-Mendoza D, Delgado-Benavides AC, Martinez-Sovero G. Composicion arborea y estructura del Bosque de Proteccion Pagaibamba, sector San Luis, Querocoto - Cajamarca. Revista
Ciencia Norandina 2020;3(1):56-67. doi:https:// doi.org/10.37518/2663-6360X2020v3n1p5

APG - Angiosperm Phylogeny Group. An update of the Angiosperm Phylogeny Group classification for the orders and families of flowering plants: APG IV. Botanical Journal of the Linnean Society 2016; 181(1), 1-20.

Asner GP, Knapp DE, Martin RE, Tupayachi R, Anderson CB, Mascaro J, Sinca F, Chadwick KD, Higgins M, Farfan W, Llactayo W, Silman MR. Targeted carbon conservation at national scales with high-resolution monitoring. Proceedings of the National Academy of Sciences 2014;111(47):E5016-E5022. doi:https:// doi.org/10.1073/pnas.141955011

Avitabile V, Herold M, Heuvelink G, Lewis S, Phillips OL, Asner GP, Armston J, Ashton PS, Banin L, Bayol N, Berry NJ, Boeckx P, de Jong B, DeVries B, Girardin C, Kearsley E, Lindsell J, Lopez -Gonzalez G, Lucas R, Malhi Y, Morel A, Mitchard E, Nagy L, Qie L, Quinones M, Ryan C, Slik J, Sunderland T, Laurin G, Gatti R, Valentini R, Verbeeck H, Wijaya A, Willcock S. An integrated pan-tropical biomass map using multiple reference datasets. Global Change Biology 2016;22(4):1406-1420. doi:https:// doi.org/10.1111/gcb.1313

Banda K, Delgado-Salinas A, Dexter KG, Linares-Palomino R, Oliveira-Filho A, Prado D, Pullan M, Quintana C, Riina R, Rodríguez GM. Plant diversity patterns in neotropical dry forests and their conservation implications. Science 2016;353(6306):13831387. doi:https://doi.org/10.1126/science.aaf508

Beer C, Reichstein M, Tomelleri E, Ciais P, Jung M, Carvalhais N, Rödenbeck C, Arain MA, Baldocchi D, Bonan GB, Bondeau A, Cescatti A, Lasslop G, Lindroth A, Lomas M, Luyssaert S, Margolis H, Oleson KW, Roupsard OL, Veenendaal E, Viovy N, Williams C, Woodward F, Papale D. Terrestrial gross carbon dioxide uptake: global distribution and covariation with climate. Science 2010;329(5993):834-838. doi:https://doi.org/10.1126/ science. 118498

Brand MA. Potencial de uso da biomassa florestal da caatinga, sob manejo sustentável, para geração de energia. Ciência Florestal 2017;27(1):117-127. doi:http:// dx.doi.org/10.5902/198050982645

Burnham KP, Anderson DR, Huyvaert KP. AIC model selection and multimodel inference in behavioral ecology: some background, observations, and comparisons. Behavioral Ecology and Sociobiology 2011;65(1):23-35. doi:https://doi.org/10.1007/ s00265-010-1029-

Campbell P, Comiskey J, Alonso A, Dallmeier F, Nuñez P, Beltran H, Baldeon S, Nauray W, De La Colina R, Acurio R, Udvardy S. Modified Whittaker plots as an assessment and monitoring tool for vegetation in a lowland tropical rainforest. Environmental Monitoring and Assessment 2002;76(1):19-41. doi:https:// doi.org/10.1023/A:101526472028

Chao A, Gotelli NJ, Hsieh TC, Sander EL, Ma KH, Colwell RK, Ellison AM. Rarefaction and extrapolation with Hill numbers: a framework for sampling and estimation in species diversity studies. Ecological Monographs 2014;84(1):45-67. doi:https:/ / doi.org/10.1890/13-0133.

Chave J, Coomes D, Jansen S, Lewis SL, Swenson NG, Zann AE. Towards a worldwide wood economics spectrum. Ecology Letters 2009;12(4):351-366. doi:https:// doi.org/10.1111/j.14610248.2009 .01285 .

Chave J, Réjou-Méchain M, Búrquez A, Chidumayo E, Colgan M, Delitti W, Duque A, Eid T, Fearnside P, Goodman R, Henry M, Martínez-Yrízar A, Mugasha W, Muller-Landau HC, Mencuccini M, Nelson B, Ngomanda A, Nogueira EM, Ortiz-Malavassi E, Pélissier R, Ploton P, Ryan CM, Saldarriaga JG, Vieilledent G. Improved allometric models to estimate the aboveground biomass of tropical trees. Global change biology 2014;20(10):3177-3190. doi:https://doi.org/10.1111/gcb.1262 
Chytrý M, Danihelka J, Axmanová I, Božková J, Hettenbergerová E, Li C-F, Rozbrojová Z, Sekulová L, Tichý L, Vymazalová M, Zelený D. Floristic diversity of an eastern Mediterranean dwarf shrubland: the importance of soil $\mathrm{pH}$. Journal of Vegetation Science. 2010;21(6):1125-1137. doi:https://doi.org/10.1111/ j.1654-1103.2010.01212.

Cueva E, Lozano D, Yaguana C. Efecto de la gradiente altitudinal sobre la composición florística, estructura y biomasa arbórea del bosque seco andino, Loja, Ecuador. Bosque (Valdivia) 2019:40 (3):365-378. doi:http://dx.doi.org/10.4067/S07179200201900030036

Dávila DC, Sanchez I, Vallejos J, Vallejos J, Appleton RD, Piana RP. Diversity and seasonality of birds in permanent waterholes in the Peruvian Northwest. Ornitología Neotropical 2019;30 (1):249-255 .

Delgado-Paredes GE, Vásquez-Díaz C, Tesén-Núñez F, EsquerreIbañez B, Zuñe-Da Silva F, Rojas-Idrogo C. Vegetación arbórea del Cerro Tres Puntas de Pilasca (Salas-Motupe), Lambayeque, Perú. Revista Mexicana de Ciencias Forestales 2020;11(58). doi:https://doi.org/10.29298/rmcf.v11i58.54

Díaz W, García V, Guillermo G. Valoración de la captura de carbono en el Santuario Histórico Bosque de Pómac y su incidencia en las políticas públicas medioambientales [tesis]. Universidad Nacional Pedro Ruiz Gallo; 2015.

Dufrêne M, Legendre P. Species assemblages and indicator species: the need for a flexible asymmetrical approach. Ecological Monographs 1977;67(3):345-366. doi:https://doi.org/10.1890/00129615 ( 1997 ) 067 [0345:SAAIST]2.0.CO;

Espinosa CI, De La Cruz M, Luzuriaga AL, Escudero A. Bosques tropicales secos de la región Pacífico Ecuatorial: diversidad, estructura, funcionamiento e implicaciones para la conservación. Revista Ecosistemas 2012;21(1-2).

Ferreyra R. Los tipos de vegetación de la costa peruana. Anales del Jardín Botánico de Madrid 1983;40(1):241-256.

Gentry AH. Diversity and floristics composition of Neotropical dry forests. In: Bullock SH, Mooney HA, editors. Seasonally dry tropical forests. Cambridge: Cambridge University Press; 1995. p. 146-194. doi:https://doi.org/10.1017/CBO9780511753398.00

GRL - Gobierno Regional de Lambayeque. Estudios de suelos con fines de Zonificación Ecológica Económica. Lambayeque: Oficina de Planificación, estrategia y ordenamiento territorial para el desarrollo sostenible; 2012.

Houghton RA. Aboveground forest biomass and the global carbon balance. Global Change Biology 2005;11(6):945-958. doi:https://doi.org/10.1111/j.1365-2486.2005.00955.

Jones MM, Ruokolainen K, Martinez NC, Tuomisto H. Differences in topographic and soil habitat specialization between trees and two understorey plant groups in a Costa Rican lowland rain forest. Journal of Tropical Ecology 2016;32(6):482. doi:https:// doi.org/10.1017/S026646741600041

Leal-Pinedo JM, Linares-Palomino R. Los bosques secos de la reserva de biosfera del noroeste (Perú): diversidad arbórea y estado de conservación/The dry forests of the Biosphere Reserve of Northwestern (Peru): Tree diversity and conservation status. Caldasia 2005;27(2):195-211.

Legendre P, Legendre L. Numerical ecology. Amsterdam: Elsevier; 2012.

Linares-Palomino R, Oliveira-Filho A, Pennington, R. Neotropical seasonally dry forests: diversity, endemism, and biogeography of woody plants. In: Dirzo R, Young HS, Mooney HA, Ceballos G, editors. Seasonally dry tropical forests. Washington, DC: Island Press; 2011. p. 3-21. doi:https://doi.org/10.5822/978-1-61091021-7_

López M, Koning FD, Paredes H, Benítez P. Estimación de carbono en biomasa de bosques secundarios y plantaciones forestales en el Noroccidente de Ecuador. Begleitprogramm Tropenökologie: Investigación de Bosques tropicales; 2002.
Málaga N, Giudice R, Vargas C, Rojas E, Zutta B, Quispe B, Secada L, Dourojeanni L, Leyva F. Estimación de los contenidos de carbono de la biomasa aérea en los bosques de Perú. Lima: Ministerio del Ambiente; 2014.

Marcelo-Peña JL. Vegetación leñosa, endemismos y estado de conservación en los bosques estacionalmente secos de Jaén, Perú. Revista peruana de biología 2008;15(1):43-52. doi:https:// doi.org/10.15381/rpb.v15i1.166

MINAM - Ministerio del Ambiente. Guía para el muestreo de suelos: En el marco del decreto supremo N002-2013-MINAM, Estándares de calidad ambiental (ECA) para suelo. Lima;2014.

MINAM - Ministerio del Ambiente. Mapa nacional de ecossistemas del Perú. Lima; 2019.

Moreno CE. Métodos para medir la biodiversidad. Zaragoza: M\&T-Manuales y Tesis SEA; 2001.

Mueller-Dombois D, Ellenberg H. Aims and methods of vegetation ecology. New York: John Wiley \& Sons; 2002

Muenchow J, von Wehrden H, Rodriguez EF, Rodriguez RA, Bayer F, Richter M. Woody vegetation of a Peruvian tropical dry forest along a climatic gradient depends more on soil than annual precipitation. Erdkunde 2013;241-248.

Ordoñez HR, Muñoz DA, Ballesteros W, Cabrera G, Mosquera J. Estimación de la biomasa aérea y captura de carbono en árboles dispersos en potreros con motilon silvestre (Freziera canescens) en el municipio de Pasto Nariño - Colombia. Revista de Ciencias Agrícola 2007;24(1-2):46-55.

Pallqui N, Monteagudo A, Phillips O, López-Gonzalez G, Cruz L, Galiano W, Chávez W, Vásquez R. Dinámica, biomasa aérea y composición florística en parcelas permanentes Reserva Nacional Tambopata, Madre de Dios, Perú. Revista Peruana Biologia 2014;21(3):235-242. doi:https://dx.doi.org/10.15381/ rpb.v21i3.1089

Pan Y, Birdsey RA, Fang J, Houghton RA, Kauppi PE, Kurz WA, Phillips OL, Shvidenko A, Lewis, SL, Canadell JG, CiaisP, Jackson RB, Pacala SW, Mcguire AD, Piao S, Rautiainen A, Sitch S, Hayes D. A large and persistent carbon sink in the world's forests. Science 2011;333(6045):988-933. doi:https:// doi.org/10.1126/science.120160

Poorter L, Bongers F, Aide T, Almeyda A, Balvanera P, Becknell J, Boukili V, Brancalion P, Broadbent E, Chazdon R, Craven D, Almeida-Cortez J, Cabral G, De Jong B, Denslow J, Dent D, Dewalt S, Dupuy J, Durán S, Espírito-Santo M, Fandino M, César R, Hall J, Hernandez-Stefanoni J, Jakovac C, Junqueira A, Kennard D, Letcher S, Licona J, Lohbeck M, Marín-Spiotta E, Martínez-Ramos M, Massoca P, Meave J, Mesquita R, Mora F, Muñoz R, Muscarella R, Nunes Y, Ochoa-Gaona S, Oliveira A, Orihuela-Belmonte E, Peña-Claros M, Pérez-García E, Piotto D, Powers J, Rodríguez-Velázquez J, Romero-Pérez I, Ruíz J, Saldarriaga J, Sanchez-Azofeifa A, Schwartz N, Steininger M, Swenson N, Toledo M, Uriarte M, Breugel M, Van Der Wal H, Veloso M, Vester H, Vicentini A, Vieira I, Bentos T, Williamson G, Rozendaal D. Biomass resilience of neotropical secondary forests. Nature 2016;530(7589):211-214. doi:https:/ / doi.org/10.1038/nature1651

Rasal M, Troncos-Castro J, Lizano-Durán C, Parihuamán-Granda O, Quevedo-Calle D., Rojas-Idrogo C, Delgado-Paredes GE. Características edáficas y composición florística del bosque estacionalmente seco La Menta y Timbes, Región Piura, Perú. Ecología Aplicada 2011;10(2):61-74.

Rasal-Sánchez M, Troncos-Castro J, Lizano-Duran C, ParihuamanGranada O, Quevedo-Calle D, Rojas-Idrogo C, Delgado-Paredes G. La vegetación terrestre del bosque montano de Lanchurán ( Piura-Perú). Caldasia 2012;34(1):1-24.

R Core Team. A Language and Environment for Statistical Computing. Vienna: R Foundation for Statistical Computing; 2020.

Rezende V, Eisenlohr P, Vibrans A, de Oliveira-Filho A. Humidity, low temperature extremes, and space influence floristic variation 
across an insightful gradient in the Subtropical Atlantic Forest. Plant Ecology 2015;216(6):759-774. doi:https:/ / doi.org/10.1007/s11258-015-0465-

Sakate DM, Kashid DN. A deviance-based criterion for model selection in GLM. Statistics 2014;48(1):34-48. doi:https:// doi.org/10.1080/02331888.2012.70803

Sanchez M, Pedroni F, Eisenlohr PV, Oliveira-Filho AT. Changes in tree community composition and structure of Atlantic rain forest on a slope of the Serra do Mar range, southeastern Brazil, from near sea level to $1000 \mathrm{~m}$ of altitude. Flora 2013;208(3):184 196. doi:https://doi.org/10.1016/j.flora.2013.03.00

Sande MT, Poorter L, Kooistra L, Balvanera P, Thonicke K, Thompson J, Arets EJMM, Garcia NA, Jones L, Mora F, Mwampamba TH, Paar, T, Peña-Claros M. Biodiversity in species, traits, and structure determines carbon stocks and uptake in tropical forests. Biotropica 2017;49(5):593-603. https:// doi.org/10.1111/btp.1245

Shepherd GJ. Fitopac 2.1.2: Manual do usuário. Campinas, SP: Unicamp; 2010.

Slik JWF, Arroyo-Rodríguez V, Aiba S-I, Alvarez-Loayza P, Alves LF, Ashton P, Balvanera P, Bastian ML, Bellingham PJ, van den Berg E, Bernacci L, da Conceição Bispo P, Blanc L, BöhningGaese K, Boeckx P, Bongers F, Boyle B, Bradford M, Brearley FQ, Breuer-Ndoundou Hockemba M, Bunyavejchewin S, Calderado Leal Matos D, Castillo-Santiago M, Catharino ELM, Chai S-L, Chen Y, Colwell RK, Chazdon RL, Clark C, Clark DB, Clark DA, Culmsee H, Damas K, Dattaraja HS, Dauby G, Davidar P, DeWalt SJ, Doucet J-L, Duque A, Durigan G, Eichhorn KAO, Eisenlohr PV, Eler E, Ewango C, Farwig N, Feeley KJ, Ferreira L, Field R, de Oliveira Filho AT, Fletcher C, Forshed O, Franco G, Fredriksson G, Gillespie T, Gillet J-F, Amarnath G, Griffith DM, Grogan J, Gunatilleke N, Harris D, Harrison R, Hector A, Homeier J, Imai N, Itoh A, Jansen PA, Joly CA, de Jong BHJ, Kartawinata K, Kearsley E, Kelly DL, Kenfack D, Kessler M, Kitayama K, Kooyman R, Larney E, Laumonier Y, Laurance S, Laurance WF, Lawes MJ, Amaral ILd, Letcher SG, Lindsell J, Lu X, Mansor A, Marjokorpi A, Martin EH, Meilby H, Melo FPL, Metcalfe DJ, Medjibe VP, Metzger JP, Millet J, Mohandass D, Montero JC, de Morisson Valeriano M, Mugerwa B, Nagamasu H, Nilus R, Ochoa-Gaona S, Onrizal, Page N, Parolin P, Parren M, Parthasarathy N, Paudel E, Permana A, Piedade MTF, Pitman NCA, Poorter L, Poulsen AD, Poulsen J, Powers J, Prasad RC, Puyravaud J-P, Razafimahaimodison J-C, Reitsma J, dos Santos JR, Roberto Spironello W, Romero-Saltos H, Rovero F, Rozak AH, Ruokolainen K, Rutishauser E, Saiter F, Saner P, Santos BA, Santos F, Sarker SK, Satdichanh M, Schmitt CB, Schöngart J, Schulze M, Suganuma MS, Sheil D, da Silva Pinheiro E, Sist P, Stevart T, Sukumar R, Sun I-F, Sunderland T, Suresh HS, Suzuki E, Tabarelli M, Tang J, Targhetta N, Theilade I, Thomas DW, Tchouto P, Hurtado J, Valencia R, van Valkenburg JLCH, Van Do T, Vasquez R, Verbeeck H, Adekunle V, Vieira SA, Webb CO, Whitfeld T, Wich SA, Williams J, Wittmann F, Wöll H, Yang X, Adou Yao CY, Yap SL, Yoneda T, Zahawi RA, Zakaria R, Zang R, de Assis RL, Garcia Luize B, Venticinque EM. An estimate of the number of tropical tree species. Proceedings of the National Academy of Sciences 2015;112(24):7472-7477. doi:https://doi.org/10.1073/pnas.142314711

Soares CPB, de Paula Neto F, de Souza AL. Dendrometria e inventário florestal. Viçosa: UFV; 2006.

Song XS, Hansen MC, Stehman SV, Potapov PV, Tyukavina A, Vermote EF, Townshend JR. Global land change from 1982 to 2016. Nature 2018;560(7720):639-643. doi:https:// doi.org/10.1038/s41586-018-0411-

Sullivan MJ, Talbot J, Lewis SL, Phillips OL, Qie L, Begne SK, Chave J, Cuni-Sanchez A, Hubau W, Lopez-Gonzalez G. Diversity and carbon storage across the tropical forest biome. Scienti- fic Reports 2017;7(1):1-12. doi:https://doi.org/10.1038/ srep3910

Tello R, Flores J. Potencial de biomasa y carbono del bosque de la llanura aluvial del rio Nanay, Perú. Conocimiento Amazónico 2010;1(1):57-67.

ter Braak CJ, Prentice IC. A theory of gradient analysis. Advances in Ecological Research 1988;34(18):271-317. doi:https:// doi.org/10.1016/S0065-2504(08)60183-

Valentin JL. Ecologia numérica: uma introdução à análise multivariada de dados ecológicos. Rio de Janeiro: Interciência; 2000.

van der Sande MT, Poorter L, Kooistra L, Balvanera P, Thonicke K, Thompson J, Arets EJ, Garcia Alaniz N, Jones L, Mora F. Biodiversity in species, traits, and structure determines carbon stocks and uptake in tropical forests. Biotropica 2017;49(5):593603. doi:https://doi.org/10.1111/btp.1245

van der Putten WH, Bradford MA, Pernilla Brinkman E, van de Voorde TF, Veen GF. Where, when and how plant-soil feedback matters in a changing world. Functional Ecology 2016;30 (7):1109-1121. doi:https://doi.org/10.1111/1365-2435.1265

Weberbauer A. El mundo vegetal de los Andes Peruanos. Lima: Ministerio de la Agricultura; 1945.

\section{Como citar este artigo \\ How to cite this article}

(ABNT)

ZUÑE DA SILVA, F.; DÁVILA RAFFO, D. C.; ROJAS IDROGO, C.; DELGADO PAREDES,G. E. Estructura y biomasa arbórea del Refugio de Vida Silvestre Laquipampa, Lambayeque, Perú. Paubrasilia, Porto Seguro, v. 4, e0055, 2021. DOI: 10.33447/ paubrasilia.2021.e0055

(Vancouver)

Zuñe da Silva F, Dávila Raffo DC, Rojas Idrogo C, Delgado Paredes GE. Estructura y biomasa arbórea del Refugio de Vida Silvestre Laquipampa, Lambayeque, Perú. Paubrasilia 2021;4:e0055. doi:10.33447/paubrasilia.2021.e0055 